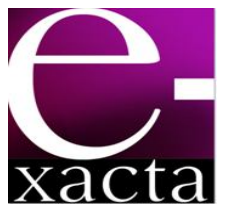

ISSN: 1984-3151

\section{Proposta PARA Gestão de Estoques EM UMA EMPRESA DE BASE TECNOLÓGICA}

\author{
METHOD FOR INVENTORY MANAGEMENT IN A TECHNOLOGY COMPANY
}

\author{
1 Bacharel em Engenharia de Produção, UFRGS, 2011. \\ Engenheira de Produção AMBEV. Porto Alegre, RS. \\ grazielehugo@gmail.com. \\ 2 Doutor em Engenharia de Produção. PPGEP/UFRGS, \\ 2013. Professor de Engenharia de Produção, DCET/UESC. \\ Ilhéus, BA. dcfettermann@gmail.com. \\ 3 Doutora em Engenharia de Produção, PPGEP/UFRGS, \\ 2004. Prof. Adjunto de Engenharia de Produção, \\ DEPROT/UFRGS. Porto Alegre, RS. \\ giovanapasa@producao.ufrgs.br.
}

Graziele Bueno Hugo'; Diego de Castro Fettermann²; Giovana Savitri Pasa ${ }^{3}$

Recebido em: 17/04/2014 - Aprovado em: 30/04/2014 - Disponibilizado em: 31/05/2014

RESUMO: A importância do gerenciamento de estoques é decorrente da busca por um melhor nível de atendimento ao cliente, fator este decisivo para a competitividade das empresas. Esse trabalho tem por objetivo a aplicação de um método para gerenciamento do estoque de produtos acabados a partir do nível de serviço em uma pequena empresa do setor eletrônico. Como principal resultado é apresentado o desenvolvimento do método realizado, a partir de um diagrama de causa e efeito dos problemas da empresa e a aplicação das nove etapas do método proposto. A aplicação do método proposto foi considerada de fácil assimilação na empresa e seus resultados permitiram sistematizar o processo de tomada de decisão quanto ao gerenciamento do estoque e minimizar os problemas de não atendimento dos pedidos por falta de produtos.

PALAVRAS-CHAVE: Gerenciamento de Estoque. Pequenas empresas. Nível de serviço.

ABSTRACT: Inventory management is an important variable for customer satisfaction and company competitiveness. This work aims to application a method for managing product inventory using service level in a small electronic industry company. The results present the method for inventory management and your application in a real case. The company's problems were analyzed on a diagram of cause and effect resulting in each phase of the proposed method. The company considered the method application easy and its results helped in the decision making process in the inventory management and also allowed a better service level.

KEYWORDS: Inventory. Small companies. Service level.

\section{INTRODUÇÃO}

A eficiente administração do estoque de peças acabadas representa um fator importante para as indústrias (BUCHER, MEISSNER; 2011), especialmente para as que atuam com operações de baixo custo (DROHOMERETSKI; FAVARETTO, 2010). A variabilidade da demanda dos pedidos e a incerteza das previsões de demanda constituem-se importantes fatores para a gestão de estoques (GIACOBO, 2003; BUCHER, MEISSNER; 2011), tendo como principal consequência o baixo nível de serviço de atendimento aos clientes (DROHOMERETSKI; FAVARETTO, 2010). Os estoques contribuem para proteger as empresas contra as incertezas de demanda, entretanto, têm por 
característica incorrer em elevados custos para a organização (SILVA; BARBOZA; MARUJO, 2008).

Altos níveis de estoques em empresas de manufatura resultam em custos de até $40 \%$ do valor dos itens estocados (BALLOU, 2001). Entretanto, a manutenção de níveis reduzidos de estoques tende a reduzir o nível de atendimento de pedidos dos clientes, resultando em perda de pedidos e prejudicando a imagem da empresa no mercado. Desta forma, os estoques devem ser gerenciados de forma equilibrada para garantir um nível de serviço adequado aos clientes e gerar lucro para a empresa (SILVER; PYKE; PETERSO, 1998; BONAFÉ; CARDOSO, 2012).

Muitas das empresas utilizam o sistema ERP (Enterprise Resource Planning) para gerenciar o sistema de estoques, entretanto esta prática costuma disponibilizar resultados não satisfatórios (BUCHER, MEISSNER; 2011). Além disso, a dificuldade na implementação dos métodos de previsão de demanda nas empresas (SOUZA et al., 2011; OZUR; PEREIRA; CORREA, 2011) afeta o gerenciamento de estoques de produtos acabados.

O estudo sobre a gestão de estoques é frequente na literatura. Kurawarwala e Matsuo (1996) desenvolveram um, para a gestão de estoques de produtos com curto ciclo de vida aplicado na indústria de computadores pessoais. Outros estudos foram realizados para controle de estoque de peças de reposição em empresas fornecedoras da indústria automobilística (LI, KUO, 2008; KUNIGAMI; OSÓRIO, 2009; BUCHER, MEISSNER, 2011). O problema de gerenciamento de estoques de peças para conserto de produtos com defeito é abordado no setor de máquinas fotográficas (TOKTAY; WEIN; ZENIOS, 2000). Apesar desses estudos abordarem a aplicação do gerenciamento de estoques, estão direcionados às empresas com maior maturidade na aplicação de técnicas e ferramentas para o gerenciamento e controle dos processos produtivos.
A partir dessa dificuldade de implementação desses métodos em empresas pequenas, o objetivo desse trabalho é aplicar um método para gerenciamento do estoque de produtos acabados a partir do nível de serviço em uma pequena empresa de base tecnológica. Este trabalho se justifica pela necessidade das pequenas empresas estruturarem o processo de tomada de decisão em relação ao planejamento da produção, níveis de estoque e atendimento ao consumidor. Atualmente, estas decisões são tomadas intuitivamente pelos gestores, sem a utilização das informações disponíveis e de um procedimento estruturado. Os métodos disponíveis na literatura são demasiadamente complexos e de difícil operacionalização pelas pequenas empresas. Dessa forma, verifica-se a necessidade de adaptação dos métodos disponíveis para a realidade, atendendo às condições de gerenciamento específicas das pequenas empresas.

Este artigo é composto por seis seções. A segunda apresenta o referencial teórico relacionado aos temas de Planejamento e Controle da Produção (PCP), previsão de demanda, estratégias de produção e gestão de estoques. A terceira seção apresenta o método de pesquisa e a empresa objeto deste estudo. O detalhamento do método proposto e os resultados obtidos da sua aplicação são apresentados na quarta seção, enquanto a quinta seção apresenta as conclusões deste trabalho.

\section{Referencial Teórico}

Nesta seção serão abordados os temas utilizados para o desenvolvimento deste trabalho. Entre esses temas estão: planejamento e controle da produção, métodos de previsão de demanda e gerenciamento de estoques. 


\subsection{Planejamento e Controle da Produção}

O PCP tem como propósito fazer a ligação entre suprimento e demanda, garantindo que os processos sejam eficazes e eficientes de forma que atendam os produtos e serviços requeridos pelos clientes (SLACK; CHAMBERS; JOHNSTON, 2009).

O PCP requer mudanças ao longo do tempo. Os planos são divididos em três níveis no horizonte de planejamento: de curto, médio e longo prazo (STEVENSON, 2001). Nos planos de longo prazo é formulado o planejamento estratégico da produção, conforme uma estimativa de vendas e a disponibilidade financeira e produtiva. Normalmente, esse planejamento é realizado pelo marketing e financeiro, atribuindo informações sobre recursos e mercados. Os planos de médio prazo são realizados pelo PCP, sendo desenvolvido o plano-mestre da produção, com base em previsões de demanda de médio prazo e pedidos já confirmados. Já os planos de curto prazo são operacionais, nos quais o PCP desenvolve a programação da produção que compreende a gestão de estoque, a liberação de ordens de compras, fabricação e montagem e, por fim, o acompanhamento e controle da produção (TUBINO, 1999). O fluxo de informações das atividades do PCP é apresentado na FIG.1.

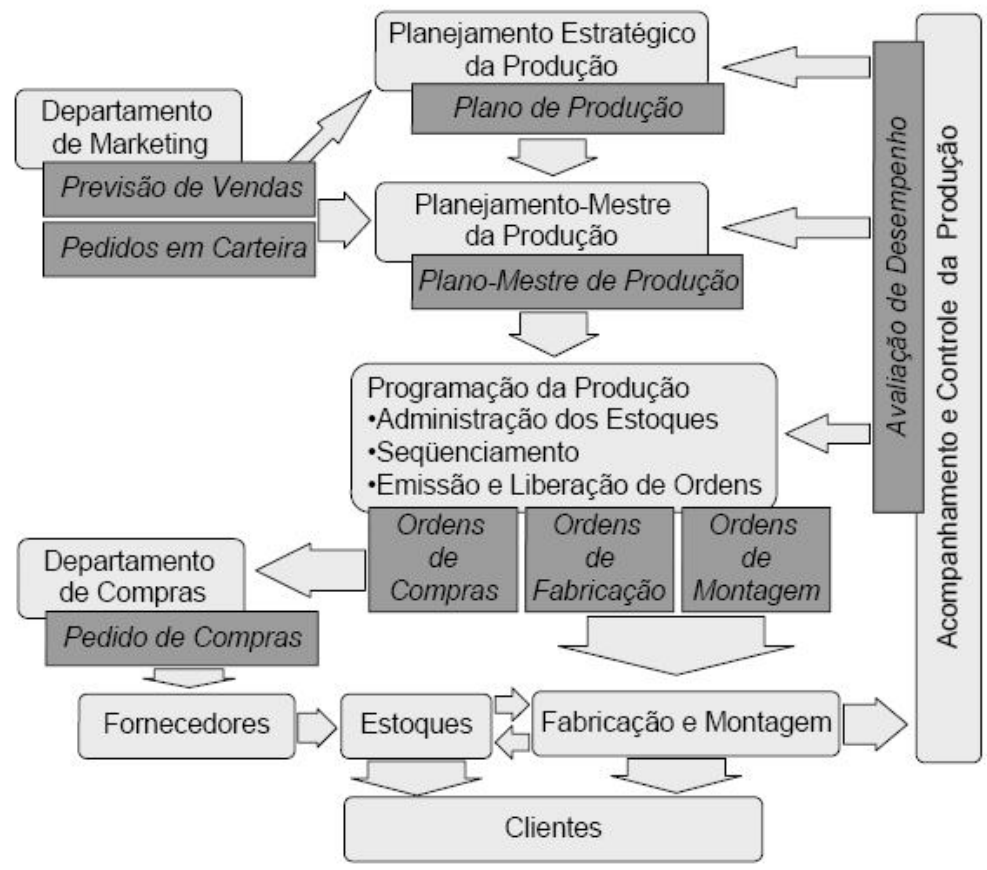

Figura 1 - Visão geral das atividades do PCP Fonte - TUBINO, 1997.

\subsection{Previsão de demANDA}

A importância da previsão de demanda supera o auxílio ao PCP e orienta a estratégia organizacional desdobrada em vários setores da empresa. As áreas funcionais necessitam de previsões futuras para estimar recursos, capacidade e políticas a serem praticadas durante certo período (LEMOS, 2006; FLORES, 2007). Dessa forma, a tomada de decisão referente à gestão de estoque está baseada principalmente na previsão de demanda (ELSAYED; BOUCHER, 1994). 
Conforme Corrêa e Corrêa (2008), um dos principais erros cometidos pelas empresas consiste em definir o quanto necessita vender para atingir os objetivos financeiros e confundir esses dados com a demanda. Previsão é um processo metodológico que estima uma demanda futura baseando-se em modelos estatísticos ou subjetivos (QUEIROZ, 2003), sendo sua realização decisiva para determinar os recursos necessários para as operações da empresa (MAKRIDAKIS; WHEELWRIGHT; HYNDMAN, 1998)

Segundo Elsayed e Boucher, (1994), qualquer previsão de demanda segue a mesma lógica: utilizar os dados do passado para prever o futuro. Para tratar essas informações existem três abordagens complementares: técnicas de previsão qualitativas, quantitativas ou a mistura de ambas (WERNER, RIBEIRO, 2003). As técnicas qualitativas são subjetivas, admitem fatores de intuição e experiência para formular opiniões que determinem a previsão. Por outro lado, as técnicas quantitativas são objetivas e baseadas em dados históricos projetados para o futuro (PELLEGRINI; FOGLIATTO, 2000; CORRÊA; CORRÊA, 2008).

As técnicas quantitativas se desdobram em métodos causais, que utilizam dados históricos sobre variáveis independentes, e análise de séries temporais, que se baseia em dados históricos para projetar a demanda futura e analisar tendências e padrões de sazonalidade (KRAJEWSKI; RITZMAN; MALHOTRA, 2008). A FIG. 2 representa um resumo dos métodos de previsão de demanda.

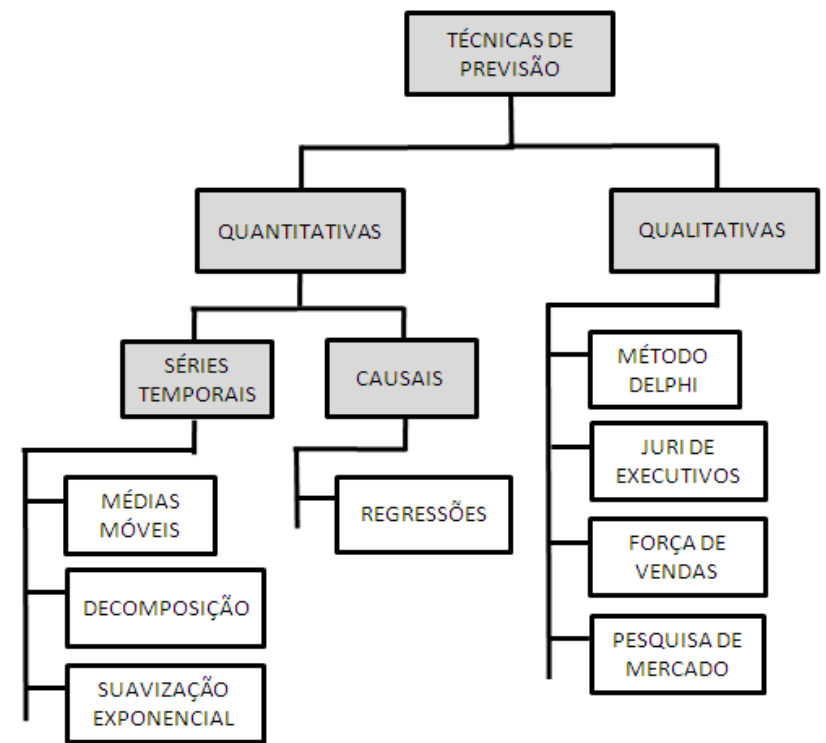

Figura 2 - Métodos de previsão de demanda Fonte - CORRÊA; CORRÊA, 2008.

\subsection{ESTRATÉGIAS DE PRODUÇÃO}

Segundo Donk, Soman e Gaalman (2000), a classificação dos produtos é o primeiro passo da análise de demanda. Os autores ainda afirmam que a classificação $A B C$ é muito simplista, reforçando a importância da classificação a partir das estratégias de produção Make to Order (MTO), Make to Stock (MTS), Assembly to Order (ATO) e Engineer to Order (ETO). Essas estratégias de produção são classificadas de acordo com as formas de relacionamento entre 
clientes e empresas (PIRES, 2004), sendo as quatro mais frequentes:

- MTS (Make to Stock): essa forma de interação é caracterizada pela produção para estoque, onde os produtos são padronizados e o planejamento é realizado através de previsões de demanda. A principal vantagem dessa estratégia é a rapidez da entrega do pedido.

- MTO (Make to Order): esse processo de interação inicia somente quando o cliente realiza um pedido. Os produtos são customizados ou projetados com itens já padronizados e admite-se alto nível de intervenção do cliente. A desvantagem dessa estratégia é o elevado tempo de entrega, por outro lado, a vantagem é a flexibilidade proporcionada ao cliente.

- ATO (Assemble to Order): nesse sistema há estoque de subconjuntos, peças e itens à espera do pedido do cliente. $O$ processo caracteriza-se pela montagem sob encomenda, limitando a interação do cliente, porém é mais ágil do que na forma MTO.

- ETO (Engineer to Order): é uma extensão do sistema MTO, onde os produtos são totalmente projetizados de acordo com as especificações do cliente. Desta forma, o tempo de pedido é mais longo do que na forma MTO.

\subsection{GESTÃo DE ESTOQUE}

O objetivo do gerenciamento de estoques é equilibrar a disponibilidade de produtos ao consumidor e os custos relacionados à manutenção dos estoques. O grande desafio é minimizar esses custos de estoque para cada nível de serviço almejado (BALLOU, 2006). Estoque é definido como matérias-primas, peças semi-acabadas e produtos finais que estão no sistema de produção durante um determinado tempo (ELSAYED; BOUCHER, 1994). E numa visão mais ampla, é todo recurso que se encontra durante e após o processo produtivo (produto acabado) (SLACK; CHAMBERS; JOHNSTON, 2002).

Entre os sistemas de controle de estoque se destacam o sistema de revisão continua $(Q)$ e o de revisão periódica (P). De acordo com Krajewski, Ritzman e Malhotra (2008), o sistema de revisão contínua (Q), também chamado de sistema de ponto de reposição, faz revisões do estoque continuamente, a cada retirada de um produto para determinar a necessidade de colocar um novo pedido. Quando a posição do estoque alcança o ponto de reposição, que é o nível mínimo, uma quantidade fixa $(Q)$ do item é colocada em pedido. Em casos onde a demanda é incerta são utilizados estoques de segurança e assim, o ponto de reposição segue a seguinte fórmula: $P R=$ demanda média durante o lead time + estoque de segurança.

Para determinar o estoque de segurança é preciso definir o nível de serviço, que está relacionado ao risco da falta do estoque e pode ser definido como a probabilidade da demanda não exceder o estoque disponível durante o lead time (STEVENSON, 2001). Corrêa e Corrêa (2008) ainda acrescentam a necessidade de quantificar as incertezas da demanda. Para isso, assume-se que a demanda real apresenta um comportamento segundo a distribuição normal, e a partir disso, encontra-se a demanda média durante o lead time $(\mu)$ e o desvio padrão ( $\alpha$ ). Por fim, considerase que o estoque de segurança é expresso como:

$$
E_{\text {seg }}=F S \times a \times \sqrt{ } \text { LT/PP }
$$

onde FS é o fator de segurança, LT é o lead time e PP é a periodicidade à qual se refere o desvio padrão (CORRÊA; CORRÊA, 2008).

O sistema de revisão periódica $(\mathrm{P})$, segundo Krajewski, Ritzman e Malhotra (2008), faz a revisão do estoque periodicamente, sendo que um pedido novo é sempre colocado após cada revisão e o tempo entre os pedidos é fixo, portanto somente o tamanho do lote $(Q)$ pode ser alterado. O gerenciamento do sistema $\mathrm{P}$ considera a tomada de duas decisões: 0 
intervalo de tempo entre as revisões $\mathrm{P}$ e a meta de estoque $\mathrm{T}$.

As principais vantagens do sistema de revisão periódica $(\mathrm{P})$ são o baixo custo de pedido, pois os pedidos de vários itens são realizados uma única vez, e a diminuição de revisões do estoque, devido à fixação de um período. Já o sistema de revisão contínua (Q) tem como vantagens o tamanho dos lotes fixos, facilitando a programação da produção e, o tamanho do estoque de segurança, menor (KRAJEWSKI; RITZMAN; MALHOTRA, 2008).

\section{MÉTODO DE PESQUISA}

Este estudo é classificado em relação à sua natureza, como uma pesquisa aplicada, visto que seu objetivo é gerar conhecimento para uma aplicação prática. Além disso, possui uma abordagem predominantemente quantitativa, pois utiliza, como fonte de dados, informações numéricas. $O$ objetivo desta pesquisa é classificado como exploratório, em razão de proporcionar maior familiaridade com o problema e torná-lo mais explicito (SILVA; MENEZES, 2001). A questão da pesquisa que orientou este trabalho consiste em: Como gerenciar o estoque de acordo com um nível de serviço pré-definido? Em razão desta pesquisa procurar responder questões do tipo "como", recomenda-se a utilização de um estudo aprofundado de um caso, característica esta que indica a aplicação de um estudo de caso (DREJER; GUDMUNDSSON, 2002).

\subsection{A EMPRESA}

O presente trabalho foi desenvolvido em uma pequena empresa do segmento eletrônico, mais especificamente, no ramo de equipamentos de monitoramento e controle de energia elétrica. A atual política da empresa favorece o desenvolvimento de novas tecnologias e novos produtos. O foco em inovação é sustentado pelas boas diretrizes do setor de marketing e pelo setor de P\&D, que é estruturado com diversos engenheiros especializados. Porém, devido ao grande crescimento no setor de equipamentos eletrônicos relacionados à energia, destacado por ABINEE (2011), a empresa tem necessidade de aprimorar o planejamento operacional para viabilizar o crescimento da demanda.

Este aumento da demanda por produtos acabados tem resultado em problemas na gestão das operações da empresa. São frequentes as alterações no plano mestre de produção, resultando em perdas no processo produtivo. Além disto, são comuns os atrasos nas entregas e inclusive são registrados casos de perdas de pedidos por falta de produtos acabados para a entrega. Por esta razão, este trabalho está direcionado para minimizar este problema, gerenciando o sistema de estoque de produtos acabados da empresa. Para isto, se propõe desenvolver um método para gerenciamento do estoque de produtos acabados a partir do nível de serviço e aplicá-lo no portfólio de produtos da empresa.

\subsection{MÉtodo de tRabalho}

O desenvolvimento do estudo partiu do problema da empresa, que é o baixo nível de serviço oferecido aos clientes, que resulta em perda de pedidos por falta de produtos para entrega. Desta forma, foi estabelecida uma relação de causa e efeito sobre cada um dos problemas, que resultou no diagrama apresentado na FIG 3.

A construção do método foi realizada de forma a minimizar cada um dos problemas identificados. Desta forma, foram desenvolvidas uma ou mais etapas do método para solucionar cada problema identificado no diagrama de causa e efeito. 


\section{Problemas}

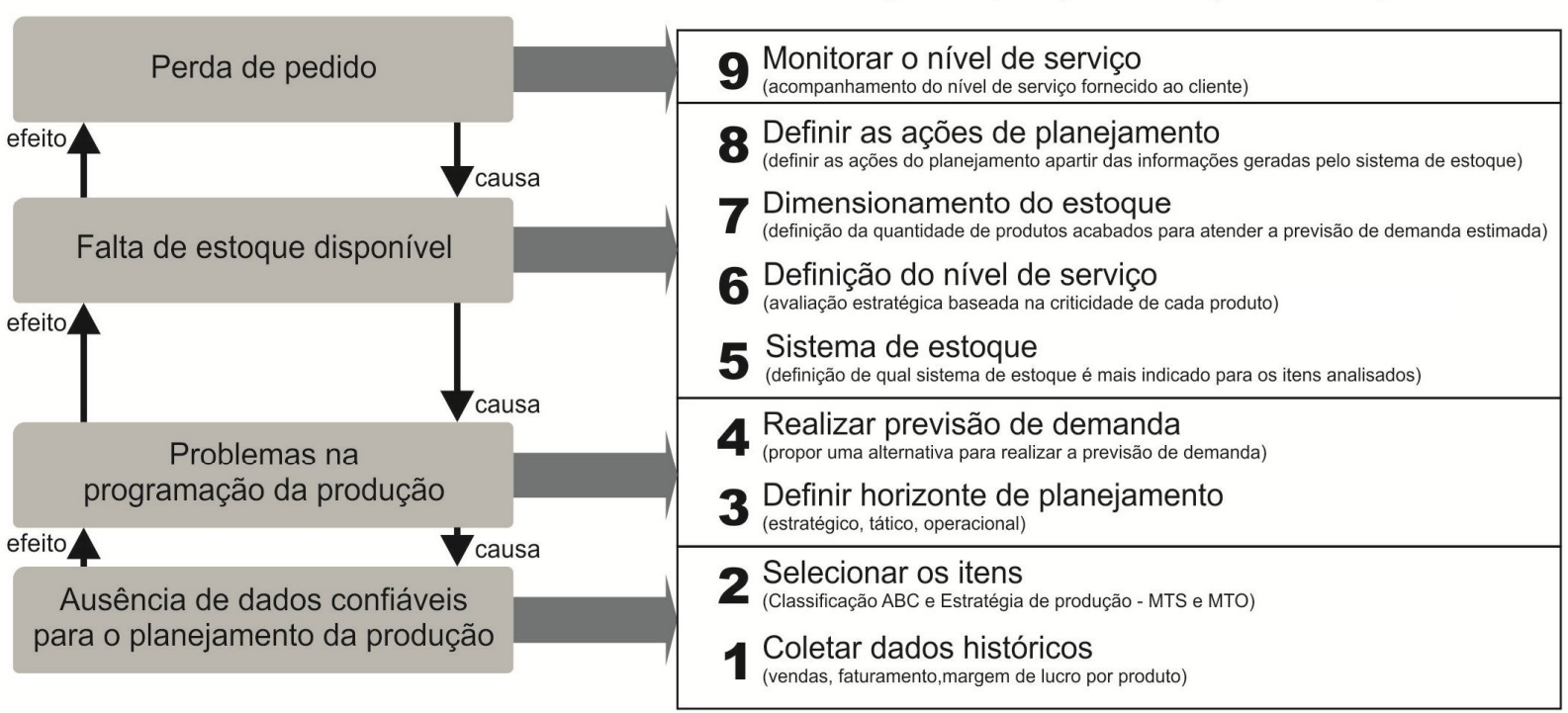

Figura 3 - Relação de causa e efeitos dos problemas e sua relação com o método proposto Fonte - Elaborado pelos autores

\section{Ações propostas (método)}

\section{RESULTADOS}

A presente seção apresenta, inicialmente, o desenvolvimento do método proposto e, a seguir, a sua aplicação em nove etapas.

\subsection{DeSENVOLVIMENTO DO MÉTOdO}

A partir da análise de causa e efeito dos problemas identificados foi desenvolvido um método de trabalho, dividido em etapas e atividades que tornam o processo aplicável. Foram estipuladas nove etapas, a saber: (i) coletar dados históricos, (ii) selecionar os itens, (iii) definir horizonte de planejamento, (iv) realizar previsão de demanda, (v) definir sistema de estoque, (vi) definir nível de serviço desejado, (vii) dimensionar estoque, (viii) definir as ações do planejamento de produção e por fim, (ix) monitorar o nível de serviço atingido. A FIG. 4 apresenta as etapas do método desenvolvido. 
sistemática proposta. Esta seleção está baseada nos tipos de estratégia de produção dos produtos (MTS/MTO) e na classificação ABC. Somente serão analisados os produtos fabricados para estoque (MTS), aqueles que são customizados e produzidos de acordo com a estratégia MTO não serão incluídos nesta análise. Entre os produtos MTS da empresa, serão selecionados os mais representativos no faturamento. Esta seleção obedece ao critério de Pareto, selecionando os produtos do tipo "A", que normalmente representam cerca de $20 \%$ do portfólio e $80 \%$ do faturamento.

$\mathrm{Na}$ terceira etapa é definido o horizonte de planejamento (iii), aplicado ao objetivo desse trabalho. Ele será proposto de acordo com o nível de planejamento do PCP e com as atividades que englobam esse horizonte. $O$ horizonte de planejamento delimita os métodos de previsão a serem utilizados.

$\mathrm{Na}$ etapa de realizar a previsão de demanda (iv) optou-se por utilizar técnicas quantitativas de previsão. Dentre elas, será escolhida uma técnica conforme o horizonte de planejamento definido anteriormente. Entre as opções para dados históricos, foram selecionados os modelos de suavização exponencial por serem amplamente utilizados devido a sua simplicidade, facilidade e boa previsão (PELLEGRINI; FOGLIATTO, 2001; SANTORO, FREIRE, 2008). Será realizada uma análise sobre o comportamento da demanda e de acordo com este comportamento será selecionado o modelo mais adequado para realizar a previsão de cada produto.

A partir da etapa de previsão de demanda, será definido o sistema de estoque (v), o qual poderá ser operacionalizado por uma revisão contínua ou periódica. Durante esta etapa também é estabelecida a periodicidade da revisão do sistema proposto para dimensionamento do estoque de produtos acabados.
A definição do nível de serviço (vi) para cada produto será definida de acordo com a criticidade de cada produto, estabelecida de acordo com a margem de lucro de cada produto. Desta forma, dentre os produtos selecionados na etapa (i), terão um nível de serviço superior os que possuem uma margem de lucro maior.

A etapa de dimensionamento de estoque (vii) realiza a definição do nível de estoque seguindo a previsão de demanda analisada e a definição do nível de serviço da etapa anterior.

$\mathrm{Na}$ etapa de definir as ações do planejamento (viii) serão identificadas as informações do sistema de estoque que alimentarão o planejamento da produção.

Por fim, a etapa de monitoramento do nível do serviço (ix) que compreende o acompanhamento de dados para a verificação da eficiência desse método de trabalho e a retroalimentação de informações ao planejamento.

\subsection{APLICAÇÃO dO MÉTOdO}

\subsubsection{COLETAR DADOS HISTÓRICOS}

A primeira etapa confere a coleta de dados da empresa em estudo. A fonte de buscas dos dados é o software ERP, utilizado pela empresa (Protheus $\AA$ ). Foram levantados dados dos produtos da empresa (73) referentes a: nome, código, a quantidade vendida, quantidade faturada, custo da matéria-prima, preço médio de venda e o faturamento. Os dados apurados compreendem os anos de 2009 e 2010 e até setembro de 2011, totalizando em 33 meses.

As quantidades vendidas e faturadas foram verificadas mensalmente, o custo da matéria-prima representa o custo médio anual do item e o faturamento foi um resultado acumulado do período estudado. A partir da coleta desses dados foi possível calcular o preço e 
lucro médio de cada item. Estas informações foram reunidas para os 73 diferentes produtos da empresa para o ano de 2011.

\subsubsection{SELECIONAR OS ITENS}

$\mathrm{Na}$ segunda etapa foi realizada a seleção dos produtos para o estudo, a seleção se baseou no método $A B C$ e na classificação de acordo com a estratégia de produção utilizada (Make to Stock, Make to Order e Engineer to Order).

Conforme os requisitos deste estudo, a seleção inicia com a seleção dos produtos com a estratégia Make to Stock. A partir dessa seleção prévia, foram destacados 39 produtos. Dentre esses produtos, para evidenciar os $20 \%$ dos produtos mais representativos para a empresa foi utilizado o critério de rentabilidade, ou seja, os produtos que mais geram lucro. A partir desse critério foi elaborado 0 indicador de rentabilidade que é a multiplicação da lucratividade de cada produto pela quantidade faturada anual. Posteriormente, foram selecionados $20 \%$ dos produtos do portfólio mais rentáveis, correspondendo a 7 produtos, que equivalem a $41 \%$ do faturamento total da empresa com a estratégia MTS. Os produtos selecionados são apresentados na TAB. 1.

Tabela 1 - Demonstrativo dos produtos selecionados

\begin{tabular}{|l|c|c|ll|}
\hline PRODUTO & CODIGO & ESTRATIEGA & WCRO'QUANIDADE \\
\hline P-600 & 7920050 & MTS & R $\$$ & $307.396,60$ \\
\hline P-600 & 7920094 & MTS & R $\$$ & $259.615,46$ \\
\hline Smart Cap 200-12 & 7810016 & MTS & R $\$$ & $217.822,23$ \\
\hline PowerCap 485 & 7840005 & MTS & R $\$$ & $200.554,62$ \\
\hline Smart Cap 485-12 & 7900001 & MTS & R $\$$ & $150.223,24$ \\
\hline P-300 & 7920040 & MTS & R $\$$ & $148.150,72$ \\
\hline Smart Cap 200-06 & 7810019 & MTS & R $\$$ & $112.145,68$ \\
\hline \multicolumn{4}{|c}{ Fonte - Elaborado pelos autores } \\
\hline
\end{tabular}

\subsubsection{DEFINIR HORIZONTE DE PLANEJAMENTO}

$\mathrm{Na}$ terceira etapa, o horizonte de planejamento foi definido de forma conceitual a partir das características da empresa e do objetivo deste estudo. Como este trabalho compreende atividades de âmbito operacional como planejamento da produção, gestão de estoque, sequenciamento e emissões de ordens de produção verifica-se a classificação do horizonte de planejamento em curto prazo. Algumas outras características da empresa dificultam um horizonte de planejamento mais longo, como o pequeno porte e a elevada variedade de produtos. Seus produtos ainda têm por característica possuir um reduzido ciclo de vida no mercado, dificultando ações de planejamento a médio e longo prazo. Para tanto, o horizonte de planejamento definido foi de três meses, com intervalo de tempo (time buckets) mensal. Desta forma, cada previsão de demanda contempla três meses futuros, possibilitando ajustes e uma melhor interpretação do mercado nas quatro previsões anuais.

\subsubsection{REALIZAR PREVISÃO DE DEMANDA}

$\mathrm{Na}$ quarta etapa é realizada a análise dos dados históricos de vendas dos produtos selecionados. Os dados históricos são analisados graficamente, auxiliando na identificação de padrões, tendências e sazonalidades na série temporal. A seleção do melhor método de previsão de demanda passa por uma análise sobre o comportamento da série temporal que pode ser representada por quatro diferentes padrões: média, sazonalidade, cíclico e tendência (MAKRIDAKIS; WHEELWRIGHT; HYNDMAN, 1998). Os padrões da série temporal obtidos para seis produtos são apresentados na FIG 5. 


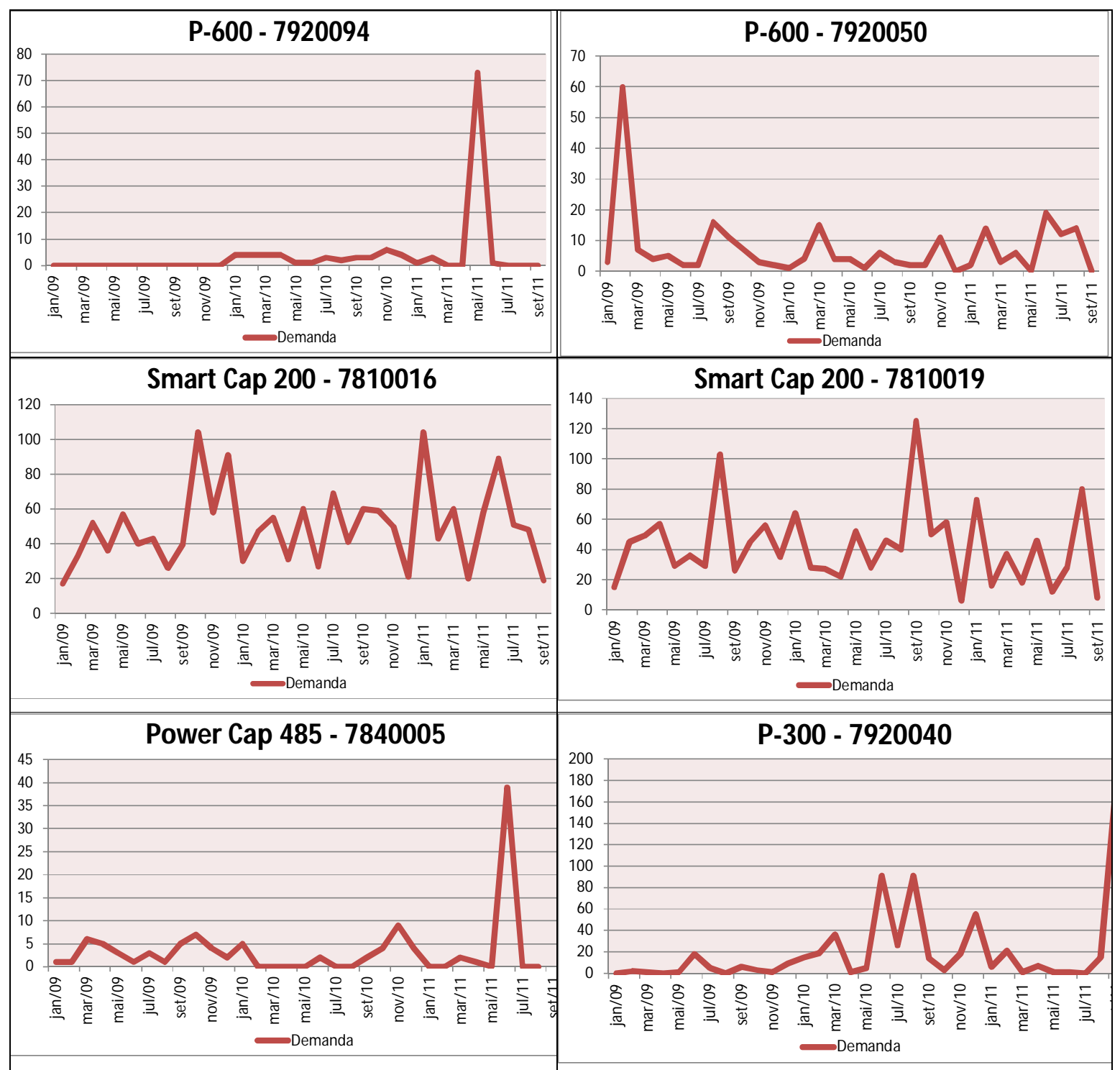

Figura 5 - Análise da série temporal dos produtos Fonte - Elaborado pelos autores

Conforme a análise obtida, alguns produtos, como o 7920094 e o 7920040, apresentam uma quantidade de dados muito restrita, pois foram lançados no mercado em menos de dois anos. Além disso, todos os produtos demonstram uma demanda irregular, que também denominada "lumpy", que se caracteriza pelo alto nível de variabilidade, demonstrando picos de demanda seguidos de períodos de demanda baixa ou nula (SANTOS; RODRIGUES, 2006). O padrão de demanda irregular ocorre em casos que a demanda é intermitente, com elevado grau de incerteza e, particularmente, difíceis de prever (LEMOS, 2006).
Como forma de estabelecer uma previsão de demanda, este trabalho segue a recomendação da gerência da empresa, obtendo a previsão de demanda a partir da média dos últimos 12 meses. A partir da obtenção de séries históricas com mais dados pretende-se implementar na empresa um sistema de previsão, baseado na suavização exponencial. A seleção do modelo de suavização exponencial dependerá do comportamento da série temporal. Em séries que se mantêm constantes sobre um nível médio, a suavização exponencial simples tende a dar melhores resultados. Para séries com tendências 
lineares o modelo de Holt tende a ser o mais indicado, enquanto que séries temporais com sazonalidade o modelos aditivo ou multiplicativo de Winters são os mais indicados (MAKRIDAKIS; WHEELWRIGHT; HYNDMAN, 1998). Para a implementação desses modelos pretende-se aplicar as formas de cálculo apresentadas por Makridakis, Wheelwright e Hyndman (1998)

\subsubsection{DEFINIR SISTEMA DE ESTOQUE}

Os produtos selecionados têm por característica uma baixa quantidade de unidades vendidas e a empresa não dispõe de um sistema confiável para monitoramento contínuo dos níveis de estoque. Os produtos monitorados também possuem demanda irregular, por esta razão um sistema que não fixar o tamanho do lote de produção tende a ser mais recomendado para aplicar na empresa. Em razão destas características, foi decidido utilizar um sistema de Revisão Periódica para gestão dos estoques.

A FIG. 6 representa o Sistema de Revisão Periódica para uma demanda aleatória. Nesse sistema, o intervalo de proteção é a soma do intervalo de revisão $(P)$ e o lead time (L), e o tamanho do pedido (Q) a ser colocado é a diferença entre o nível alvo de estoque (T) e o nível de estoque atual (NE).

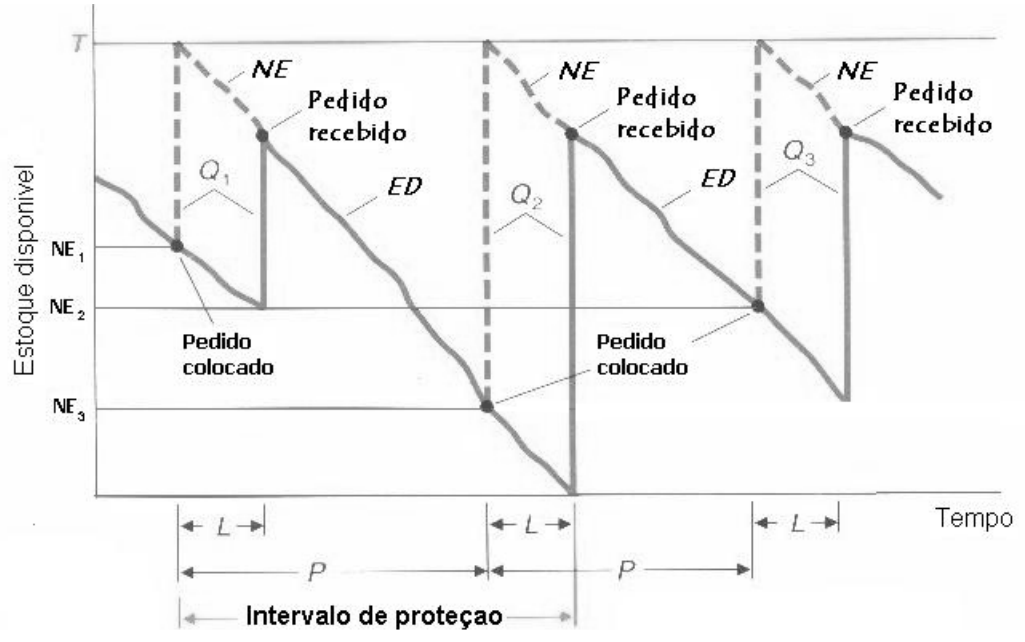

Figura 6 - Sistema de revisão periódica

Fonte - KRAJEWSKI; RITZMAN; MALHOTRA, 2008.

Ao desenvolver esse sistema é necessário estimar o intervalo de revisão $(P)$ desejado, o lead time dos produtos e obter informações como:

- Demanda durante o intervalo de proteção: $\mu(p+l)$

- Desvio-padrão durante intervalo de proteção: $\sigma(p+l)$ $=\sigma^{*} \sqrt{ }(p+l)$

- Nível de serviço desejado para cada produto, e sua probabilidade: $\mathrm{z}$

- Estoque de segurança: $E S=z^{*} \sigma(p+l)$

- Nível alvo: $T=\mu(p+l)+E S$
A empresa tem por característica produzir diversos produtos que atendem diferentes estratégias, tais como ETO, MTO e MTS. Os produtos MTS, abordados neste trabalho têm por característica uma programação de produção para períodos de maior folga no sistema produtivo. O PCP da empresa costuma programar a produção destes itens a cada quatro semanas, a fim de reduzir perdas de set up de máquinas. Outra razão para esta programação mensal é a curva de aprendizado dos operadores, visto que nem todos são treinados e costumam demorar em 
atingir níveis satisfatórios de produção para o item. Por estas razões, estes produtos MTS costumam ter uma programação de produção mensal dentro da empresa. Como forma de reduzir o impacto no PCP, esta proposta mantém o intervalo de revisão $(P)$ de quatro semanas já utilizado na empresa. Esta meta pode ser revisada e reduzida, assim que o sistema proposto seja melhor testado e confiável. Uma vez verificada a necessidade de fabricação desses produtos, esta normalmente é realizada em no máximo quatro dias, resultando em 0,5 semanas.

\subsubsection{DefinIR Nível DE SERVIÇo DESEJADo}

A definição do nível de serviço foi estabelecida conforme a criticidade de cada produto, que foi mensurada através de duas variáveis: lucratividade e estratégia de vendas. A partir destes dois critérios foi estabelecida uma escala de 1 a 5 para cada fator, sendo que 1 significa baixa criticidade da variável e 5 uma alta criticidade. A TAB. 2 apresenta os critérios para a elaboração da criticidade dos produtos.

Tabela 2 - Critérios de Criticidade

\begin{tabular}{|c|c|c|c|}
\hline $\begin{array}{l}\text { Citicidade } \\
\text { chlugo }\end{array}$ & Legenda & $\begin{array}{l}\text { Oitiaidade } \\
\text { ceEstráǵgia }\end{array}$ & Legenda \\
\hline 1 & Até300\%/cdeluoro & 1 & $\begin{array}{l}\text { Prodtos commais } \\
\text { de5anos dentrado }\end{array}$ \\
\hline 2 & De300a600\% & 2 & De5a2anos \\
\hline 3 & Dewa900\% & 3 & $\begin{array}{c}\text { Proditos commenos } \\
\text { de2anos }\end{array}$ \\
\hline 4 & De900a $1200 \%$ & 4 & $\begin{array}{l}\text { Prodtos commenos } \\
\text { de2anos + sutwere }\end{array}$ \\
\hline 5 & Adinade $1200 \%$ & 5 & Proditos novos \\
\hline
\end{tabular}

Fonte - Elaborado pelos autores

A partir da classificação do produto quanto à criticidade do lucro e da estratégia é calculada a criticidade do produto, que é dada pelo resultado da multiplicação entre a criticidade de lucro e de estratégia, sendo que o valor obtido define o nível de serviço demonstrado na TAB. 3.
Tabela 3 - Criticidade relativa ao nível de serviço

\begin{tabular}{|c|c|}
\hline $\begin{array}{c}\text { Criticidade } \\
\text { do Produto }\end{array}$ & $\begin{array}{c}\text { Nível de } \\
\text { Senviço }\end{array}$ \\
\hline De 1 a5 & $90 \%$ \\
\hline De $6 a 16$ & $95 \%$ \\
\hline De 17 a 25 & $98 \%$ \\
\hline
\end{tabular}

A partir dos cálculos propostos foi possível calcular o nível de serviço desejado para cada um dos produtos. Entre os produtos selecionados foram identificados os seguintes níveis de serviço:

- Produto 7920050 - criticidade 20 e nível de serviço $98 \%$

- Produto 7920094 - criticidade 08 e nível de serviço $95 \%$

- Produto 7810016 - criticidade 02 e nível de serviço $90 \%$

- Produto 7840005 - criticidade 10 e nível de serviço $95 \%$

- Produto 7900001 - criticidade 03 e nível de serviço $90 \%$

- Produto 7920040 - criticidade 04 e nível de serviço $90 \%$

-Produto 7810019 - criticidade 02 e nível de serviço $90 \%$

\subsubsection{DiMENSIONAR ESTOQUE}

$\mathrm{Na}$ etapa de dimensionamento de estoque é definido, para cada produto, o nível alvo de estoque (T). Esse dimensionamento é realizado conforme a previsão de demanda, o sistema de estoque escolhido e o nível de serviço de cada produto.

Na TAB. 4 constam os valores obtidos na previsão de demanda, o cálculo de todas as informações 
relevantes para o sistema de estoque e, de todos os produtos.

consequentemente, o valor obtido para o nível alvo ( $T$ )

Tabela 4 - Resultados da etapa de dimensionamento de estoque

\begin{tabular}{|c|c|c|c|c|c|c|c|c|c|c|c|}
\hline CODIGO & $\begin{array}{c}\mu: \\
\text { DEMANDA } \\
\text { MÉDIA } \\
\text { EM P }\end{array}$ & $\begin{array}{c}\sigma: \\
\text { DESVIO } \\
\text { EM P }\end{array}$ & $\begin{array}{l}\text { NÍVEL DE } \\
\text { SERVIÇO }\end{array}$ & $\begin{array}{c}\text { Z: } \\
\text { PROB. }\end{array}$ & PERÍODO & $\begin{array}{l}\text { LEAD } \\
\text { TIME }\end{array}$ & $\begin{array}{c}\text { INTERVALO } \\
\text { DE PROTEÇÃO } \\
(p+1)\end{array}$ & $\begin{array}{c}\mu(p+1): \\
\text { DEMANDA } \\
\text { NO } \\
\text { INTERVALO }\end{array}$ & $\begin{array}{c}\sigma(p+1): \\
\text { DESVIO NO } \\
\text { INTERVALO }\end{array}$ & $\begin{array}{c}\text { ES: } \\
\text { ESTOQUE DE } \\
\text { SEGURANÇA }\end{array}$ & $\begin{array}{l}\text { T: NÍVEL } \\
\text { ALVO DE } \\
\text { ESTOQUE }\end{array}$ \\
\hline 7920050 & 6,92 & 6,72 & $98 \%$ & 2,05 & 4 SEM & $0,5 \mathrm{SEM}$ & 4,5 SEM & 7,78 & 14,26 & 29,28 & 37,06 \\
\hline 7920094 & 3,25 & 1,42 & $95 \%$ & 1,64 & 4 SEM & $0,5 \mathrm{SEM}$ & 4,5 SEM & 3,66 & 3,02 & 4,96 & 8,62 \\
\hline 7810016 & 51,83 & 24,83 & $90 \%$ & 1,28 & 4 SEM & $0,5 \mathrm{SEM}$ & $4,5 \mathrm{SEM}$ & 58,31 & 52,67 & 67,50 & 125,81 \\
\hline 7840005 & 3,58 & 2,07 & $95 \%$ & 1,64 & 4 SEM & $0,5 \mathrm{SEM}$ & $4,5 \mathrm{SEM}$ & 4,03 & 4,38 & 7,21 & 11,24 \\
\hline 7900001 & 14,75 & 6,47 & $90 \%$ & 1,28 & 4 SEM & $0,5 \mathrm{SEM}$ & $4,5 \mathrm{SEM}$ & 16,59 & 13,72 & 17,59 & 34,18 \\
\hline 7920040 & 11,83 & 15,46 & $90 \%$ & 1,28 & 4 SEM & $0,5 \mathrm{SEM}$ & 4,5 SEM & 13,31 & 32,79 & 42,02 & 55,33 \\
\hline 7810019 & 36,00 & 25,47 & $90 \%$ & 1,28 & $4 \mathrm{SEM}$ & $0,5 \mathrm{SEM}$ & $4,5 \mathrm{SEM}$ & 40,50 & 54,02 & 69,23 & 109,73 \\
\hline
\end{tabular}

\subsubsection{DEFINIR AS AÇÕES DO PLANEJAMENTO DE PRODUÇÃo}

Após a realização das etapas anteriores, o planejamento da produção é redefinido a cada quatro semanas com as seguintes informações: (i) número de produtos presentes no estoque (NE) e o (ii) nível alvo (T) de cada produto para a colocação dos pedidos $(Q)$. As previsões de demanda, a classificação da criticidade e o dimensionamento do nível alvo do estoque ( $T$ ) são realizados trimestralmente alterando as informações do sistema de estoque. A partir dessas informações, o planejamento de estoque alimenta um banco de dados que é utilizado na próxima etapa do método proposto.

\subsubsection{MONITORAR O NÍVEL DE SERVIÇO ATINGIDO}

Nesta etapa é realizado o monitoramento do nível de serviço atingido para os clientes. Isto significa que serão verificadas quantas vezes, entre os produtos com estratégia MTS monitorados, houve alguma falta de produtos acabados para serem entregues aos clientes. Este monitoramento contribui para identificar a eficácia do processo proposto através de um indicador do nível de serviço oferecido ao cliente.
Contudo, além do esforço de manter os estoques capazes de garantir o atendimento ao cliente, é fundamental realizar melhorias no processo para reduzir os estoques e, consequentemente, os custos de guarda. Para isso, é importante aprimorar a coleta de dados históricos e as técnicas de previsão de demanda, etapas que no início da aplicação do método são prejudicadas devido à falta de dados.

\section{CONSIDERAÇõES FINAIS}

O presente método de trabalho, apesar de aplicado na empresa, o impacto de sua implementação ainda não pôde ser amplamente mensurado. No período de seis meses não foram verificados registros de falta de produtos acabados para os produtos gerenciados a partir do método proposto. Sua aplicação, entretanto, tem contribuído na organização do processo de tomada de decisão em relação ao nível de estoque e a programação da produção.

A aplicação do método proposto demonstra-se prática e objetiva, visto que segue as nove etapas e em cada 
uma está bem definido as decisões que devem ser tomadas e a ordem de necessidade de cada uma. A não complexidade do método favorece sua aplicabilidade em empresas pequenas, pois ele não exige a formulação de cálculos complexos e difíceis, contribuindo para uma maior acessibilidade aos profissionais da área de produção.

Os resultados obtidos a partir do dimensionamento do estoque apresentam um elevado nível de estoques sugerido pelo sistema de gestão proposto. Isso se justifica pela variabilidade da demanda e também pelo nível de serviço estipulado. Porém, são previstas ações de melhoria no sentido de reduzir os custos dos altos níveis de estoque propostos, tais como: aprimorar a coleta de dados e as técnicas de previsão de demanda, referentes às etapas (i) e (iv). Como sugestões de trabalhos futuros propõem-se 0 monitoramento dos resultados obtidos em um período maior e sua aplicação nos demais produtos do portfólio da empresa.

\section{REFERÊNCIAS}

ABINEE - ASSOCIAÇÃO BRASILEIRA DA INDÚSTRIA ELÉTRICA E ELETRÔNICA. Desempenho Setorial. Disponível em: http://www.abinee.org.br/abinee/decon/decon15.htm. Acesso em 09 de junho de 2011.

BALLOU, R. H. Gerenciamento da Cadeia de Suprimento/Logística Empresarial. 5 ed. Porto Alegre: Bookman, 2006.

BONAFÉ, P.; CARDOSO, A.A. Gestão Lean da Linha de Montagem de uma Indústria Multinacional do ramo Petrolífero. E-xacta, v.5, n.2, p.111-126, 2012.

\section{BUCHER, D.; MEISSNER, D. Spare Parts Safety} Stock Planning in the German Automotive Industry - a Case Study. Disponível em http://www.meiss.com. Fevereiro, 2001.

CORRÊA H. L; CORRÊA C. A. Administração de Produção e Operações. 2 ed. São Paulo: Atlas, 2008.

DONK, D. P.; SOMAN, C. A.; GAALMAN, G. A decision Aid For Make-To-Order or Make-To-Stock Classification in Food Processing Industries.

Proceedings...: EurOMA International Conference on Operations and Global Competitiveness, 19-22, Budapest, Hungary, 2005.

DREJER, A.; GUDMUNDSSON, A. Towards multiple product development. Technovation, v.11, p.733-745, 2002.

DROHOMERETSKI, E.; FAVARETTO, F. Um levantamento das causas e efeitos da falta de acuracidade nos estoques: um estudo exploratório.

Revista Gestão Industrial, v.6, n.2, p.142-158, 2010.
ELSAYED, E. A.; BOUCHER, T. O. Analysis and Control of Production Systems. 2. ed. Upper Saddle River, NJ: Prentice - Hall, 1994.

FLORES, J. H. F. Aplicação de Redes Neurais Artificiais à Previsão de Vendas de Máquinas Agrícolas. Anais... Encontro Nacional de Engenharia De Produção (ENEGEP), 11, Foz do Iguaçu, 2007. Foz do Iguaçu: ABEPRO, 2007.

GIACOBO, F.; CERETTA, P. S. Planejamento Logístico: Uma Ferramenta para o Aprimoramento do Nível de Serviço. Anais..VI Semead, São Paulo, 2003.

KRAJEWSKI; RITZMAN; MALHOTRA, L.; Administração de Produção e Operações. 8. ed. São Paulo: Pearson, 2008.

KUNIGAMI, F.J.; OSÓRIO, W.R. Gestão no controle de estoque: um estudo de caso em montadora automobilística. Revista Gestão Industrial, v.5, n.4, p.24-41, 2009.

KURAWARWALA, A.,A.; MATSUO, H. Forecasting and Inventory Management of Short Life-Cycle Products. Operations Research, v.44, n.1, 1996.

LEMOS, F. O. Metodologia para Seleção de Métodos de Previsão de Demanda. Porto Alegre, 2006. Dissertação em Engenharia de Produção, Programa de Pós-Graduação em Engenharia de Produção- UFRGS.

LI, S.G.; KUO, X. The inventory management system for automobile spare parts in a central warehouse. Expert Systems with Applications, v.34, n.2, p.1144-1153, 2008.

MAKRIDAKIS, S.; WHEELWRIGHT, S. C; HYNDMAN, R. J. Forecasting Methods and Applications. 3. ed. New York: John Wiley \& Sons, 1998. 
OZUR, F.S.; PEREIRA, T.H.; CORREA, J.A.S. Controle de Demanda de Energia Elétrica. E-xacta, v.4, n.3, p.191-202.

PELLEGRINI, F.R.; FOGLIATTO, F.S. Passos para implantação de sistemas de previsão de demanda: técnicas e estudo de caso. Produção, v.11, n.1, p.4364, 2001.

PIRES, S. Gestão da Cadeia de Suprimentos. São Paulo: Atlas, 2004.

QUEIROZ, A. A. Método de previsão de demanda e detecção de sazonalidade para o planejamento da produção de indústrias de alimentos. Anais... Encontro Nacional de Engenharia de Produção. Ouro Preto, 2003.

SANTORO, M.C.; FREIRE, G. Análise comparativa entre modelos de estoque. Produção, v.18, n.1, p.8998, 2008.

SANTOS, A. M.; RODRIGUES, lana Araújo. Controle de estoque de materiais com diferentes padrões de demanda: estudo de caso em uma indústria química. Revista Gestão e Produção, v.13, n.2, p.223-231, 2006.

SILVA, E. L.; MENEZES, E. M.T. Metodologia da Pesquisa e Elaboração de Dissertação. 3. ed. Florianópolis: Laboratório de Ensino a Distância da UFSC, 2001.

SILVA, T.G.; BARBOZA, F.; MARUJO, L. Análise dos impactos das diferentes políticas de gestão de estoques de suprimentos em uma empresa do setor de bebidas através de simulação. Revista Gestão Industrial, v.4, n.4, p.34-75, 2008.
SILVER, E.; PYKE, D.F.; PETERSO, R. Inventory Management and Production Planning and Scheduling. John Wiley \& Sons. $3^{\circ}$ ed. New York, 1998.

SLACK, N.; CHAMBERS, S.; JOHNSTON, R. Administração da Produção. 3. ed. São Paulo: Atlas, 2009.

SOUZA, R.S.; TRIERWEILER, A.C.; WEISE, A.D.; ROCHA, R.A.; MONTEIRO, M.I. Previsão de demanda como suporte para o planejamento e controle da produção na Sigma - Indústria Eletro Eletrônica. Revista Ingepro - inovação, gestão e produção, v.3, n.4, 2011.

STEVENSON, W. J. Administração das Operações de Produção. 6. ed. Rio de Janeiro: LTC, 2001.

TOKTAY, L.B.; WEIN, L.M.; ZENIOS, S. Inventory Management of Remanufacturable Products.

Management Science, v.46, n.11, 2000.

TUBINO, D. F. A Produtividade no Chão de Fábrica. 1. ed. Porto Alegre: Bookman, 1999.

WERNER, L.; RIBEIRO; J.L.D. Previsão de demanda: uma aplicação dos modelos Box-Jenkins na área de assistência técnica de computadores pessoais.

Gestão \& Produção, v.10, n.1, p.47-67, 2003. 